und zum Alter der Erde hierauf aufmerksam gemacht. Hier wie bei all den Methoden mit Hilfe der Radioaktivität das Alter zu bestimmen, sind systematische Fehler da, die es zu hoch erscheinen lassen.

Beachtenswert ist eine Bemerknng von SoDDY, die einen oberen Grenzwert für das Alter der Erde gibt. Da nämlich das Lebensalter des Urans ein begrenztes ist, so können Mineralien, selbst wenn sie ursprünglich ganz aus metalliscbem Uran best:nden hätten, nicht über ein bestimmtes Lebensalter, - die Halbwertszeit ist, etwa 1010 Jahre, besitzen. Da man nach dem Gesetz der Molekularproportionen für viele Uranmineralien angenähert den wahren maximalen Urangehalt angeben kann, so lässt sich die Grenze noch enger ziehen, etwa $10^{9}$ $=1000$ Millionen Jahre; sie ist natürlich höher als das wahre aus dem Heliumgehalt angenähert ermittelte Alter.

Wenn wir die Ergebnisse der in beiden Abschnitten dargelegten Berechnungen zusammenfassen, so lässt sich mit Sicherheit so viol sagen: Die seit dem Anfang des Algonkian verstrichene Zeit ist, wie aus den Abkïhlungsberechnungen folgt, grösser als 30 Mill. Jahre und, wie aus den Radioaktivitätsmessungen folgt, kleiner als 600 Mill. Jahre. Dem Ref. scheint ein Wert von 100-200 Mill. Jahren am wahrscheislichsten.

\title{
Die Ergebnisse der neueren Erdbebenforschung in bezug auf die physikalische Beschaffenheit des Erdinnern.
}

\author{
Von F. Pockels (Heidelberg).
}

\section{Literatur.}

1. E. Wiechert. Über die Massenverteilung im Innern der Erde. Göttinger Nachr. 1897.

2. W. Schweydar. Beitrag z. Bestimmung des Starrheitskoeffizienten der Erde. Beitr. z. Geophys. 9, S. 41, 1907.

3. E. WIECHERT. Die Erdbebenforechung, ihre Hilfsmittel und ihre Resultate für die Geophysik (Vortr. a. d. Naturf.-Vers. zu Dresden 1907. Physik. Zeit. schr. 9, S. 36, 1908).

4. H. ARnoul, Die Erdbewegung während des 1. Vorläufers eines Erdbebens. Dissert. Göttingen 1909. Beitr. z. Geophys. 10, S. 269, 1909.

5. W. Líska. Mitt. d. Erdbebenkommission. Wien, N. F. 14, 1903.

6. - - Verwendung der Erdbebenbeob. z Erforschung. d. Erdinnern. Wiener Sitzungsber. 113, II a, S. 739. 1904.

7. H. Benndorf. Fortpflanzung der Erdbebenwellen. Wiener Sitzungsber. 114, II a, S. 1407, 1905 und 115 II a, S. 941, 1906.

8. E. Wiecherr. Theoretisches über die Ausbreitung der Erdbebenwellen. Gött. Nachr, 1907, S. 1.

9. K. Zöppritz. Laufzeitkurven. Gött. Nachr. 1907, S. 115.

10. K. Zöppritz u. L. Geiger. Über Erdbebenwellen III. Gött. Nachr. 1909. S. 400 .

11. J. Micne. The velocity of propagation of earth quake vibrations. Brit. Assoc. Rep. 1903. S. 84. 
12. R. D. Oxdham. Constitution of the interior of the Earth. Quart. journ. of geol. soc. 62, S. 456.1906.

13. K. G Kхотт. Seismic radiations. Edinb. Proc. 28, S. 217. 1908.

\section{§1. Verschiedene Anhaltspunkte für Sehlüsse über das Erd.} in nere.

Bis vor wenigen Jahrzehnten waren die Anhaltspunkte für sichere Schlüsse über die physikalische Natur des Erdinnern in Tiefen, die grösser sind als einige Kilometer, sehr beschränkt. Fs kamen in Betracht nur die Gravitationswirkung der Erde selbst, wie sie auf ihrer Oberfläche beobachtet wird (also die Grösse und Verteilung der Schwere auf der Erdoberfläche), und die Einwirkung anderer Weltkörper (Sonne und Mond) auf den Erdkörper, die sich einmal in einer Beeinflussung der Rotationsbewegung des ganzen Frdkörpers (Präzession und Nutation) zweitens in dessen Gezeitendeformation (Ebbe und H'lut der festen Erdoberfläche) äussert. Aus diesen Erscheinungen konnte man ableiten: Die mittlere Dichtigkeit der ganzen Erdmasse, gewisse Bedingungen für die Dichteverteilung, endlich einen Durchschnittswert des, ,Starrheitskoeffizienten “ - d.h. des elastischen Widerstandes gegen Formänderungen - des ganzen Frdkörpers. Ge n a u ist nur unsere Kenntnis der mittleren Dichte der Erde $(5,527)$, die sich auf die sehr exakten Messungen der Gravitationskonstante einerseits, der Schwere an der Erdoberfläche andererseits gründet. Aber schon über die Dichteverteilung im Erdinnern kann mit Sicherheit nur qualitativ geschlossen werden, dass eine Dichtigkeitszunahme gegen den Erdmittelpunkt hin vorhanden ist; um jedoch deren Gesetz abzuleiten, reichen die oben erwähnten Beobachtungstatsachen bei weitem nicht ans, und man musste Hypothesen hinzunehmen. Von diesen ist die plausibelste wohl die von WIEcherT (1), dass die Erde aus einem nahezu homogenen Metallkern, umgeben von einem ebensolchen Gesteinsmantel, bestehe; dann lässt sich aus der mittleren und oberflächlichen Dichte in Verbindung mit der Abplattung der Radius und die Dichte des Metallkerns berechnen. Ersterer ergibt sich zu etwa ${ }^{4} / 5$ des Erdradius und letztere ungefähr gleich 8, was in gewissem Grade als Bestätigung der aus anderen Grïnden wahrscheinlichen Annahme gelten kann, dass der Erdkern in der Hauptsache aus Eisen bestehe. Man wird sich indessen, trotz dieses plausiblen Resultates, gegenüber der Wiechertschen Annahme von der "Zweiteilung" der Erdmasse dem Bedenken nicht verschliessen können, dass die Dichtigkeit des Steinmantels doch wahrscheinlich nicht konstant ist, sondern stetig mit der Tiefe zunimmt, und dass der Übergang desselben in den Metallkern wohl schwerlich ein sprunghafter sein wird.

Die Beobachtungen über die Gezeitendeformationen des festen Erdkörpers, sowie über die Polschwankungen, aus denen man die durchschnittliche Starrheit der Erde berechnen kann, sind sehr subtiler Art und daher keiner grossen relativen Genauigkeit fähig. Da man aber auf jenen beiden voneinander nnabhängigen Wegen zu nahe dem gleichen Resultat gelangt ist (2), so darf dieses, dass näm. lich die mittlere Starrheit der Erde ungefähr derjenigen des Stahles gleichkommt, als ziemlich sichergestellt gelten. Doch wird der Wert dieser Erkenntnis dadurch stark vermindert, dass es sich um einen Mittelwert handelt, der selbst keine reale Bedeutung als physikalische Konstante des Erdkörpers besitzt, ja sogar nicht einmal einen zuverlässigen Schluss auf den Aggregatzustand des Hrdinnern gestattet. Denn auch eine Flüssigkeit von sehr grosser Zähigkeit 
könnte das hier in Betracht kommende Verbalten gegenüber periodisch veränderlichen Kräften zeigen, während andererseits die vollständige Nachgiebigkeit, die sie gegenüber konstanten oder sehr langsam veränderlichen Kräften zeigen würde, anch beim Erdkörper wirklich vorliegt (Anpassung der Abplattung an die gegenwärtige Rotationsgeschwindigkeit, isostatisches Gleichgewicht der Kontinente). Erwähnt sei noch, dass neuerdings auch (von ScHWEYDaR, [2]) der Versuch gemacht ist, unter Annahme der zweigeteilten Erde die Starrheit von Kern und Mantel gesondert zu berechnen, wobei sich die erstere etwa dreimal grösser, die letztere fast 8 mal kleiner als diejenige von Stahl ergab, ein Ergebnis, das für das Vorhandensein einer zäbflüssigen Magmaschicht im Gesteinsmantel zu sprechen scheint.

Bei der vorstehend gekennzeichneten Sachlage ist es nun höchst erfreulich, dass die verfeinerten Beobachtungsmethoden der Seismologie einen neuen Weg eröffnet haben, um Kenntnisse über das Erdinnere - zunächst über dessen elastische Eigenschaften - zu erlangen; und zwar einen Weg, der den früheren oben erwähnten deshalb sehr überlegen ist, weil er nicht nur Mittelwerte für die ganze Erde Jiefert, sondern bei genügender Vervielfältigung des Beobachtungsmaterials es prinzipiell ermöglicht, über die elastischen Eigenschaften jeder beliebigen TiefenschichtAufschluss zuerhalten. In welcher Weise die seismischen Beobachtungen zur Lösung dieser Aufgabe dienen können, und welche Resultate sie bisher geliefert haben, soll im folgenden kurz dargelegt werden.

\section{\$2. Entwickelung und Ziele der Seismologie.}

$\mathrm{Da}$ die Seismologie naturgemäss in denjenigen Ländern entstanden ist, welche häufig von starken Erdbeben heimgesucht werden, so waren ihre Beobachtangsmethoden zunächst auf "Nahbeben" eingerichtet und dienten dazu, die Zeit und Richtung der Erdbebenstösse zu registrieren. Es ist aber klar, dass durch solche Beobachtungen in der Nähe des Erschütterungsherdes keine Aufschlüsse über die Beschaffenheit der tieferen Teile des Erdinnern gewonnen werden können; ja, selbst den nächstliegenden $\mathrm{Zweck}$, die genaue Lage, insbesondere Tiefe des Erdbebenherdes zu bestimmen, haben sie bisher nicht in befriedigender Weise erfüllen können. Es kommen also für die Erforschung des Erdiunern lediglich die Beobachtungen von "Fernbeben“, d. h. solehen, deren Herd in einigen Tausend Kilometer Entfernung vom Beobachtungsort liegt, in Betracht, - Beobachtungen, die erst durch die in den letzten Jahrzehnten konstrajerten feinen Seismometer ermöglicht wurden, da es sich hier um Bodenbewegungen handelt, die oft nur Bruchteile eines Millimeters erreichen. Die Registrierungen der Seismographen liefern einmal gewisse Anfschlüsse über die Art der Bodenbewegung am Beobachtungsort und damit über die Art der Wellen, durch welche die Erschütterung in ihren einzelnen Phasen fortgepflanzt wird; sodann liefern sie den Zeitpunkt des Beginns der Erschütterung oder bestimmter Phasen derselben, und durch die Vergleichung dieser an verschiedenen Stationen beobachteten Zeiten dann die nötigen Anhaltspunkte zur Berechnung des Weges und der Geschwindigkeit der Erdbebenwellen im Erdinnern.

Hierin also liegt in erster Linie der grosse Wert der Fernbeben-Beobachtungen für die Geophysik, nicht, wie es nach der Zeitungsberichterstattung wohl Manchem scheinen mag, in der bloss statistischen Feststellung der Zeit und des Ortes der Erdbeben. Eine solche Statistik kann sich $z$ war auch von Nutzen erweisen, aber 
mehr in geologischer als geophysikalischer Hinsicht, z. B. zur Aufdeckung von Beziehungen zwischen der Lage der Erdbebenherde und dem geologischen Bau der äusseren Erdrinde; aber hierfür werden im allgemeinen Nahbeobachtungen besser verwertbar sein als Fernbeobachtungen, da letztere nur eine sehr rohe Bestimmung des „Epizentrums" - d. h. der über dem Krdbebenherd gelegenen Stelle der Erdoberfläche - gestatten und so gat wie gar keinen Aufschluss über die Tiefe des Herdes liefern.

\section{Beschaffenheit der Seismogramme.}

Für die hier zu betrachtenden Folgerungen brancht auf die Konstruktion and Wirkungsweise der Seismographen nicht näher eiugegangen zu werden ${ }^{1}$ ). Nur daran sei erinnert, dass jeder Seismograph im Prinzip einem Pendel vergleichbar ist, dessen träge Masse schnellen Bewegungen des das Pendel tragenden Gestelles nicht sogleich folgt, und welches daher bei Erderschütterungen relativ zu seiner Umgebung Bewegungen ausführt, die mittelst mechanischer oder photographischer Übertragung in starker Vergrösserung auf einem durch eiu Uhrwerk bewegten. Papierstreifen aufgezeichnet werden. $\mathrm{Tm}$ ein vollständiges Bild der Bodenbewegung zu erhalten, muss je eine solche Aufzeichnung für zwei zueinander senkrechte horizontale Komponenten und für die vertikale Komponente der Bewegung gemacht werd̉en, wozu mindestens zwei Instrumente: ein „Horizontalseismograph" und ein "Vertikalseismograph" erforderlich sind. Aber auch für jede einzelne Komponente ist das durch das Seismogramm gelieferte Bild des zeitlichen Verlaufes der betreffenden Bodenbewegang weit davon entfernt ein ähnliches Abbild zu sein, und zwar infolge der Eigenschwingungen, in die das Seismometer gerät. Wie schwierig es ist, aus einem Seismogramm die ihm zugrunde liegende wirkliche Bodenbewegung abzaleiten, hat kürzlich H. ArnoLd (4) gezeigt. Dies beeinträchtigt aber nicht die Verwendbarkeit der Seismogramme für die Ableitung des Weges und der Geschwindigkeit der Erdbebenwellen durch das Erdinnere: denn hierfür kommt es nur auf die genaue Feststellung der Zeit. punkte an, zu denen die erste Erschütterung, und weiterhin bestimmte Phasen der Erschütterung, an den verschiedenen Stationen eintreffen

Der typische Verlauf eines Fernbeben-Diagrammes ist etwa folgender. (Siehe Fig. 1, welche ein Seismogramm vom 19. III. 1906 nach WiecherT darstellt). Nachdem der Seismograph vorher in Ruhe war, also auf dem bewegten Papier eine glatte gerade Linie aufzeichnete, beginnt mit einer plötzlichen Ausweichung eine Reihenfolge nnregelmässiger Wellen von meist sehr kleiner Amplitude und kurzen Perioden (von 1-10 Sekunden): die "ersten Vorläafer". Diese dauern je nach der Entfernung des Erdbebens, eirige - eventuell bis über 10 - Minuten. Dann beginnen mit einem meistens plötzlichen Einsatz etwas stärkere Oszillationen mit längeren Perioden (von 10 Sekunden und darüber); diese sich über einen Zeitraum, der etwas kürzer ist als die Dauer der ersten Vorläufer, erstreckenden Schwingungen werden als „zweite Vorläufer" bezeichnet. Ihnen folgen die „Hauptwellen", welche mit langsamen Schwingungen (von 20 bis 60 Sekunden

1) Eine ausführliche Theorie der Seismographen gab Wiechert, Göttinger Abhandlungen N.F. 2, Nr. 1, 1903 , eine kürzere, übersichtliche Darstellung derselbe in Phys. Zeitschr. 9, S. 36, 1908, sowie H. Arvol. in seiner Dissertation (Göttingen 1909, auch in Beitr. z. Geophys. 10, S. 269, 1909). 
Schwingungsdauer) beginnen, dann etwas kürzere Perioden aufweisen und dabei die grössten Amplituden erreichen, welche diejenigen der Vorläufer weit übertreffen. Dann nehmen die Amplituden allmählich ab, jedoch zeitweilig wjeder stärker werdend, während die Perioden ziemlich konstant bleiben. Diese Schlussphase der B $\theta$ wegung (Nachläufer) kann oft stundenlang andauern. Es ist nun vor allem wichtig, dass die Zeitdauer der ersten und zweiten Vorläufer gar nicht von der speziellen Natur des Erdbebens (etwa von dessen Dauer im Epizentrum), sondern lediglich von der Entfernung des Herdes, bezw, des Epizentrums, abhängt, derart, dass man aus jenen Zeiten nit ziemlicher Sicherbeit sogleich diese Entfernung $\Delta$ (längs der Erdoberfläche gemessen) angeben kann. Man hat hierfür verschiedene empirische Formeln aufgestellt; z. B. ist nach Laska (5) die Dauer der ersten Vorläufer in Minuten minus 1 gleich der Entfernung 1 längs der Erdoberfläche in Tausenden Kilometer, oder es ist die Dauer des ganzen Vorbebens gleich $3 \Delta$. Besser noch benutzt man aber zur Bestimmung von $\Delta$ eine graphische Darstellung. (Es ist kIar, dass man, wenn man in dieser Weise die Entfernung $\Delta$ des Epizentrums von zwei Siationen ermittelt hat, damit die Lage des Epizentrums als einen der Schnitt. punkte zweier Kreise auf der Erdoberfläche kennt, und dass das Seismogramm einer dritien Station ausrojeht, um diese Lage eindentio zu bestimmen. In der Tat ist dies der gewöbnliche Weg, wie man das Epizentrum von Fernbeben ermittelt).

Die nächste, uns hier interessierende Frage ist nun die: was bedenten jene drei Einsätze eines Furnbebens? Die Beobachtungstatsachen, an die man sich bei dieser Deutung zunächst zu halten hat, sind folgende:

1. Die Fortpflanzungszeit $T$ (vom Epizentrum bis zum Beobachtungsort) der Hauptwellen ist proportional der längs der Erdoberfläche gemessenen Entfernung $\Delta$; die "Laufzeitkurve", welche man erhält, wenn man $\Delta$ als Abszisse, T als Ordinate aufträgt, ist also für die Hauptwellen eine Gerade.

2. Für die ersten und zweiten Vorläufer wächst $T$ langsamer als $\Delta$; ihre Laufzeitkurven sind keine Geraden, haben aber für die ersten nnd $z$ weiten Vorlāufer einen ähnlichen Verlauf.

Aus 1. ist zu schliessen, dass die Hauptwellen längs der Oberfläche laufen, oder doch sich in den äussersten Schichten der Erdrinde fortpflanzen; denn nur dann kann sich die längs der Oberfäche gemessene Geschwindigkeit konstant, d. h. unabhängig von der Herddistanz, ergeben.

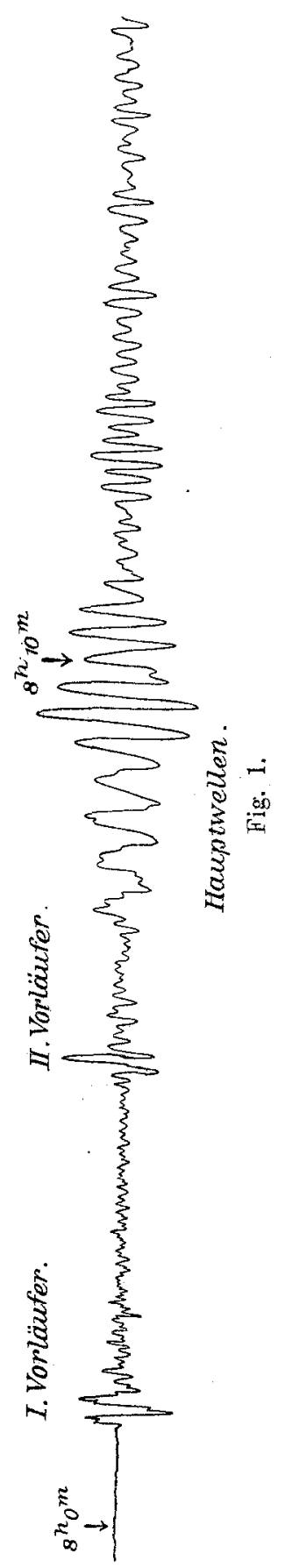


Umgekehrt folgt aus der Tatsache 2, dass die Vorläufer sich nicht längs der Oberfläche, sondern durch das Erdinnere fortpflanzen. Die Fortpflanzung erfolgt aber nicht in geradlinigen Strahlen mit konstanter Geschwindigkeit; denn in diesem Fall müsste, da die Länge der Sehne vom Erschütterungszentrum zum Beobachtangsort $=2 \mathrm{R} \sin \Delta / 2 \mathrm{R}$ ist, die Gleichung der Laufzeitkurve sein :

$$
\mathrm{T}=\text { konst. . } \sin \frac{\Delta}{2 \mathrm{R}} \quad(\mathrm{R}=\text { Erdradius }),
$$

was weder für die ersten noch für die zweiten Vorlänfer zutrifft. Wir kommen auf die Gestalt der Erdbebenstrahlen im Erdinnern unten noch zurück. - Eine Bestätigung findet die obige Folgerung für die ersten Vorläufer auch in der Vergleichung der gleichzeitig aufgezeichneten horizontalen und vertikalen Bewegungskomponenten. Es spricht nämlich alles dafür, dass die ersten Vorläufer long itudinal schwingende Wellen sind. Dann muss bej ihnen die horizontale Bewegungsrichtung in der Richtung des grössten Kreises durch Epizentrum und Beobachtungsort liegen, was die Beobachtungen an Horizontalseismographen bestätigen. Ferner muss die vertikale Kumponente im Vexgleich zur horizontalen um so grösser werden, je weiter entfernt der Bebenherd ist, weil mit der Entfernung die Neigung, unter welcher der durch das Erdinnere gehende Strahl die Erdoberfläche trifft, ebenfalls zunimmt; und dies ist in der Tat zufolge der Vergleichung der bei den ersten Vorläufern erhaltenen Vertikal- und Horizontal. Seismogramme der Fall.

Bezüglich der Schwingungsart der zweiten Vorläufer waren die Ansichten der Seismologen lange geteilt; manche (z. B. LÁsKA (6)) deuteten sie als reflektierte Longitudinalwellen, Benndorf (7) und WrecherT (2) dagegen als Transversalwellen, die sich direkt durch das Erdinnere fortpflanzen; letztere Ansicht ist jetzt wohl allgemein angenommen. Danach müssen in der zweiten Vorstörung, umgekehrt wie in der ersten, die horizontalen Bewegungen um so mehr überwiegen, je grösser $\Delta$ ist.

Was endlich die Schwingungsart der Hauptwellen betrifft, so sind nach Wi echert hier zwei Wellenarten zu unterscheiden: rein transversale mit horizontaler Schwingungsichtung, und solche, deren Schwingung elliptisch ist in der Vertikalebene durch die Fortpflanzungsrichtung. Erstere haben etwas grössere Geschwindigkeit. Auf ihre besondere Bedentung lsommen wir im nächsten Paragraphen zarück.

\section{\$ 4. Elastische Wellen in isotropen festen Körpern.}

Um die vorstehenden Sätze zu erläutern und die im folgenden zu besprechenden Schlüsse verständlich zu machen, müssen wir hier einiges über die Fortpflanzung von Schwingungen in festen Körpern einscbalten.

Wir beschränken uns dabei auf js otrope Körper, da man bisher in den Untersuchungen über Erdbebenwellen die Erde zwar als inhomogen, aber als isotrop behandelt hat, - eine Voraussetzung, die allerdings für die äusserste Erdrinde schwerlich zutrifft, mit grösserer Wahrscheinlichkeit jedoch für die unter sehr hohem Druck stehenden Tiefen, auf die es hier hauptsächlich ankommt. Im Innern eines isotropen festen Körpers also können sich zwei Arten von elastischen Wellen, bei denen die Schwingungsamplitude in der Wellenebene konstant ist, mit bestimmten, für alle Richtungen gleichen Geschwindigkeiten unabhängig voneinander fortpflanzen. Für die einen, deren Schwingungen longitu - 
dinal, also (wie die Schallwellen in Flüssigkeiten) mit Volumänderungen des Mediums verbunden sind, ist die Fortpflanzungsgeschwindigkeit:

$$
\text { 1. } \mathrm{va}=\sqrt{\frac{\mathrm{a}^{2}}{\varepsilon}},
$$

wo $\mathrm{a}^{2}$ den elastischen Widerstand des Körpers gegen einseitige Kompression (d. h. den einseitigen Druck dividiert durch die lineare Kontraktion in seiner Richtung), und $\varepsilon$ seine Dichtigkeit bedeutet. In den Wellen der zweiten Art erfolgen die Schwingungen transversal, d. h. senkrecht zur Fortpflanzungsrichtung, und sind daher nicht von Dichteänderungen, aber von Formänderungen (Schiebungen) der Volumelemente begleitet; dementsprechend hängt ihre Fortpflanzungsgeschwindigkeit von dem elastischen Widerstande $b^{*}$ gegen Formänderungen oder der "Starrheit" (englisch, rigidity ${ }^{\text {) }} a b$, und zwar ist sie

$$
\text { 2. } \mathrm{vb}=\sqrt{\frac{\mathrm{b}^{2}}{\varepsilon}} \text {. }
$$

Die beiden hier anftretenden elastischen Konstanten $\mathrm{a}^{2}$ und $\mathrm{b}^{2}$ hängen mit denjenigen Konstanten, durch welche man gewöhnlich das elastische Verhalten isotroper Körper charakterisiert: dem Elastizitätsmodul E (= Dehnungswiderstand eines freien Stabes vom Querschnitt 1) und dem Verhältnis $\mu$ der Querkontraktion zur Längsdilatation eines, einseitigem Zuge unterworfenen Stabes (dem "Poissonschen Koeffizient"), durch folgende Beziehungen zusammen:

$$
\begin{aligned}
& \text { 3. } \quad \mathrm{E}=\frac{3 \mathrm{a}^{2}-4 b^{2}}{\mathrm{a}^{2}-b^{2}} \cdot b^{2}, \\
& \text { 4. } \mu=\frac{\frac{1}{2} \mathrm{a}^{2}-b^{2}}{\mathrm{a}^{2}-b^{2}} .
\end{aligned}
$$

Ferner ist die kubische Kompressibilität

$$
\text { 5. } \frac{1}{\mathrm{~K}}=\frac{1}{\mathrm{a}^{2}-\frac{4}{3} \mathrm{~b}^{2}} \text {. }
$$

Für Körper, deren Querkontraktionskoeffizient den Wert $\frac{1}{4}$ hätte (welchem der wirkliche Wert für die meisten festen Körper, insbesondere auch für die die Erdrinde hauptsächlich zusammensetzenden Gesteine, nahekommt), wäre

$$
\text { 6. } \mathbf{a}^{2}=3 b^{2} \text {. }
$$

Wira an irgend einer Stelle im Innern des Körpers eine Erschütterung erzeugt, so werden im allgemeinen beide vorstehend gekennzeichneten Wellen entstehen und sich von dem gemeinschaftlichen Zentrum aus unabhängig voneinander ausbreiten (als $\mathrm{K} \mathbf{u}$ g elwellen, soweit der Körper als homogen betrachtet werden kann); dabei trennen sie sich infolge ihrer verschiedenen Geschwindigkeit (nach Gl. 1,2 u. 6 ist annähernd $v_{a}=v_{b} \cdot \sqrt{3}$ ) um so weiter voneinander, je grösser die Entfernung vom Erregungs-Zentrum ist. Da nun, wie oben ausgeführt, die ersten Vorlänferwellen der Erdbeben als Jongitudinale, die zweiten Vorläufer als transversale Wellen aufzufassen sind, so ist leicht verständlich, weshalb die Zwischenzeit zwischen dem Eintreffen der ersten and der zweiten Vorläufer mit der Entfernung des Bebenzentrums wächst.

Die gegenseitige Unabhängigkeit der longitudinalen (a-) und transversalen (b-) Wellen hört auf, wenn sie eine Unstetigkeitsfläche, insbesondere die freeie Oberfläche des Körpers treffen; $d$. h. es entstehen dann aus einer einfallenden $a$ - oder $b$-Welle im allgerneinen $z$ wei $r e f l e k t i e r t e$ Wellen verschiedener Art. Die Verhältnisse hierbei sind im einzelnen folgende (8): 
Aus einer a-Welle entsteht eine unter gleichem Winkel reflektierte a-Welle und eine unter steilerem Winkel reflektierte $b$-Welle; letztere schwingt in der Einfallsebene.

Aus einer senkrecht zur Einfallsebene schwingenden b-Welle entsteht nur eine gleichartige, unter glejchen Winkel reflektierte Welle.

Aus einer in der Einfallsebene schwingenden b-Welle entsteht bei Reflexion, wenn der Einfallswinkel kleiner als arcsin b/a ist, eine gleichartige b-Welle und eine a-Welle, letztere unter einem vom Einfallswinkel verschiedenen Winkel; wenn abex der Einfallswinkel > aresin b/a ist, so wird nur eine b-Welle reflektiert, aber ausserdem entsteht eine neue Art von Wellen, die längs der Oberfläche hinlaufen, n Ellipsen parallel zur Einfallsebene schwingen, und ein Analogon besitzen bei der totalen Reflexion des Lichtes.

Die ein oder mehrere Male an der Erdoberfläche reflektierten Wellen, sowie die bei der Reflexion entstehenden Oberflächenwellen haben bis zum Beobachtungsort längere Wege zu durchlaufen, als die direkt fortgepflanzten, und werden also erst einige Zeit nach Beginn des ersten bezw. zweiten Vorläufers eintreffen. Solche neue Einsätze der Schwingungsbewegung, die sich auf reflektierte Wellen zarück* führen lassen, sind nicht selten in den Seismogrammen erkennbar.

Im übrigen ist klar, dass die reflektierten Wellen überhaupt wesentlich dazu beitragen werden, die Seismogramme kompliziert za gestalten und in die Länge zu ziehen.

Oberf $\downarrow$ ächenwellen von ähnlicher Art wie die oben erwähnten, bei der "totalen" Reflexion von b-Wellen entstehenden, können nun auch selbständic (d. b. ohne Verbindung mit einer aus dem Innern kommenden einfallenden Welle) existieren, wie Lord RAYletGH znerst nachgewiesen hat. In diesen Wellen schwingen die Teilchen ebenfalls in Ellipsen, deren Ebene senkrecht zur Oberfläche und parallel zur Fortpflanzungsrichtung, und deren grosse Achse senkrecht zur Obetfiäche ist ${ }^{1}$ ); die Schwingungsweite nimmt dabei von der Oberfläche ans nach unten nach einer geometrischen Progression ab, derart, dass sie in einer T'iefe gleich der halben Wellenlänge nur noch ungefähr den 4. Teil von derjenigen in der Oberfläche beträgt, und dass sich also auch bei den sehr langen derartigen Wellen der Erdbeben die ganze Bewegung auf eine relativ zum Erdradius sehr dünne Oberflächenschicht der Erde (von weniger als $100 \mathrm{~km}$ Dicke) beschränkt.

Ferner besitzen diese "RAYLEIGH-Wellen" eine bestimmte, d. b. nur von den Elastizitätskonstanten abhängige Fortpflanzungsgeschwindigkeit $\nabla_{R}$, die im Falle $\mathrm{a}^{2}=3 \mathrm{~b}^{2}$ etwas kleiner als diejenige der Transversalwellen ist ( $V_{\mathrm{R}}$ etwa $=$ 9/10 vh). Solche RAYLEIGMsche Oberflächenwellen werden dann entstehen,

1) Obgleich also in den Hauptwellen eine vertikale Schwingungskomponente vorhanden ist, und ibre Schwingungsart den Meereswellen qualitativ vergleichbar ist, sind sie doch von keiner merklichen N eigungs-Änderung der Erdoberfiäche begleitet, wie man leicht einsiebt, wenn man die ausserordentliche Kleinheit der Schwingungsamplitude im Verhältnis zur Wellenlänge berïcksichtigt. Da man früher, selbst für Fernbeben, "Neigungswellen" annahm, hat SCHLÜTer (Beitr. z. Geophys. 1903) das Nichtvorhandensein messbarer Neigungen bei Fernbeben mittelst eines besonders konstruierten, n u $r$ auf Neigungen reagierenden Seismographen $($ "Klinograph") von sehr grosser Empfincilichkeit auch experimentell nachgewiesen. 
wenn das Erschütterungszentrum nahe der Oberfläche des festen Körpers liegt ${ }^{1}$. Dieser Fall ist aber bei den Erdbeben gegeben, und es ist daher za erwarten, dass diese Art von Wellen in den seismischen "Hauptwellen * vertreten sind. Dies wird bestätigt durch die konstante Oberflächengeschwindigkeit der letzteren, sowie durch den Wert dieser Geschwindigkeit $(3,5 \mathrm{~km} / \mathrm{sec}$.) im Verhältnis zu demjenigen von relativ flach verlaufenden $b$-Wellen.

Wie schon oben bemerkt wurde, sind aber in den Hauptwellen der Erdbeben nach WIECHERT (8) auch rein transversale, zur Erdoberfläche parallele Schwingungen vorhanden. Diese können nicht denł RAYLEIGH - Wellen angehören und sind als Oberflächenwellen nur möglich, wenn sie sich in einer auch nach unten von einer frei beweglichen Oberfläche begrenzten, dünnen Kugelschale fortpflanzen, - also nicht auf einer einheitlichen festen Kugel.

Die Existenz dieser transversalen Oberflächenwellen würde also zu dem Schluss nötigen, dass eine verhältnismässig dünne äussere feste Erdrinde auf einer flüssigen Magmaunterlage gleichsam schwimmt - eine Vorstellung, die auch mit bekannten Folgerungen aus den Schweremessungen in bestem Einklange steht, andererseits aber dem Einwande ausgesetzt ist, dass dann nicht transversale Wellen durch das Erdinnere hindurch gehen könnten, wie es in den 2. Vorlăufern (die ja allerdings viel schwächer sind als die Hauptwellen) doch der Fall ist. Nun kann man zwar nach Beobachtungen an Flüssigkeiten annehmen, dass bei grosser Zähigkeit - wie sie der Magmaschicht zweifellos zuzuschreiben wäre sich auch transversale Schwingungen von hinreichend kurzer Periode durch diese fortpflanzen könnten; allein dann dürfte man wieder die Grenzfläche der festen Kruste gegen die Magmaschicht nicht als in horizontaler Richtung frei beweglich betrachten. Mindestens müsste man erwarten, dass die fraglichen transversalen Oberflächenwellen besonders stark gedämpft wären, was durchaus nicht der Fall ist, da sie ja zu den Hauptwellen gehören. Man sieht also, dass die Deutung der sich längs der Erdoberfläche fortpflanzenden Hauptwellen noch beträchtlichen Schwierigkeiten begegnet.

\section{§5. Deutung vorherrschender Perioden in den Erdbebenwellen.}

Zu dem Schlusse, dass wahrscheinlich in relativ geringer Tiefe eine "flüssige" oder doch sehr nachgiebige Magmaschicht existiere, ist WIEchERT auch noch auf. einem anderen Wege gelangt. $(8, \S 15)$.

In den Seismogrammen sind, neben unregelmässigen stossartigen Bewegungen, meist lange Reihen von mehr oder weniger regelmässigen Schwingungen erkennbar, insbesondere in den Hauptwellen. Diese Schwingungen müssen von Eigen schwingungen irgendwelcher Teile der Frdrinde herrühren, die durch das Erdbeben ausgelöst sind. WrecherT ist der Ansicht, dass es sich dabei hauptsächlich um horizontale Transversal-Schwingungen handelt. Nun kommen in den grossen Weltbeben vorwiegend Perioden von 17 bis 18 Sekunden vor, anscheinend

1) Übrigens setzt die RAYLEIGH'sche Lösung voraus, dass die betreffenden Wellen schon sehr lange bestehen. Die Ra YLEIGH - Wellen mit der Geschwindigkeit $V_{R}$ können sich also erst allmählich bei länger dauernder Erregung herausbilden, und die ersten Oberflächenwellen müssen sich mit der Geschwindigkeit. $v_{b}$ ausbreiten. Hierdureh erklärt Wrechent die besonders langen Perioden zu. Bıginn der Hauptstörung. 
unabhängig von der geographischen Lage des Herdes. Dies veranlasst Wiechert zu der Hypothese, dass es sich in diesen Fällen um Eigenschwingungen der ganzen auf der Magmaschicht ruhenden Erdrinde (in der Umgebung des Herdes) handelt, wobei die horizontalen Schwingungen an der freien Oberfläche und an der Grenze gegen die Magmaschicht die grösste Amplitude und entgegengesetzte Phase haben, in der Mitte der ganzen Schicht dagegen verschwinden. Bei dieser "Grandschwingung" einer oben und unten freien Scholle ist also deren Dicke D gleich einer halben Wellenlänge der Transversalwellen; ist $T$ ihre Periode, so folgt demnach $D=1 / 2 \mathrm{~T} . \mathrm{vb}_{\mathrm{b}}$. Nun ist für die äussere Erdrinde durchschnittlich $\mathrm{v}_{\mathrm{b}}=4 \mathrm{~km} / \mathrm{sec}$ (siebe unten); setzt man also $\mathrm{T}=17^{1 / 2} \mathrm{sec}$ ein, so folgt

$$
\mathrm{D}=35 \mathrm{~km}
$$

als Dicke der festen Erdrinde bis zur Magmaschicht, ein Wert, den WigchERT (3) auch nach dem Betrage der geothermischen Tiefenstufe für wahrscheinlich hält. Dieser Wert von D wird allerdings von anderen für zu klein gehalten. Z. B. hat HELiverT aus den Schwerebeobachtungen in der Nähe von Küsten für die „Ausgleichsfläche ${ }^{*}$, bis za welcher die Dichtigkeitsunterschiede unter den Kontinenten und Meeren reichen, eine Tiefe von etwa $120 \mathrm{~km}$ berechnet, und auf die gleiche Grössenordnung führte die Untersuchung der Lotabweichungen in Nordamerika durch TItTMAnN und HAYFORD; diese Ausgleichsfläche würde aber nach der PratT schen Hypothese mit der unteren Grenze der festen Kontinentalmassen zusammenfallen. Inmerhin wird die WIECHERT sche Hypothese weiter zu verfolgen sein; namentlich wäre es in dieser Beziehung von Interesse, die vorherrschenden Perioden bei Erdbeben mit kontinentalen und bei solchen mit subozeanischen Herden zu vergleichen.

\section{\$6. Allgemeine Sätze über Erdbebenstrahlen im Erdinneren.}

Nach der vorstehenden, die Schwingungsperiode betreffenden Abschweifung kehren wir zu dem Hauptproblem zurück: der Ermittelung des Weges und der Geschwindigkeit der sich durch das Erdinnere fortpflanzenden Erdbebenwellen, von denen dabei nach dem oben Gesagten nur die 1. und 2. Vorläufer in Betracht kommen.

Wir erinnern hier zunächst daran, dass auf elastische Wellen gerade so wie auf Lichtwellen das Hurgess sche Prinzip anwendbar ist, wonach eine spätere Lage der Welle aus einer fräheren ableitbar ist als einhüllende Fläche aller Elementarwellen, welche sich in der $\mathrm{Z}$ wischenzeit von sämtlichen Punkten der früheren Welle aus fortgepflanzt haben. Ferner können wir in demselben Sinne, wie in der Optik, von Strahlen reden (d. h. mit diesem Ausdruck die Fortpflanzungsrichtung der einzelnen Wellenflächenelemente bezeichnen) und da wir die Erde an jeder Stelle als elastisch is o tr op voraussetzen (siehe oben S. 254), so sind die Strablen, wie in der Optik einfach brechender Medien, überall senkrecht auf den Wellenflächen. Wir haben es aber bei der Erde mit einem inhomogenen Medium zu tun, und in einem solchen sind die Strahlen im allgemeinen gekrümmt, wie sich ohne weiteres aus dem Huxgens schen Prinzip ergibt. Nun kann bei der Untersuchung der Erdbebenstrahlen die geringe Abplattung der Erde vernachlässigt werden, und ferner wird es zulässig sein, die physikalische Beschaffenheit, wenigstens in einiger Tiefe unter der Oberfläche, als nur vom Radius ablängig anzusehen (für Abweichungen hiervon liegen jedenfalls bisher keine Anzeichen vor). Dann aber haben wir es mit einem "kugelförmig geschichteten" isotropen 
Medium zu tun; es soll dabei angenommen werden, dass die ${ }_{n}$ Schichten" stetig ineinander übergehen, so dass keine Reflexionen und Brechungen stattfinden. (Wenn sich nachher zeigt, dass Unstetigkeitsflächen vorhanden sind, so kann man doch die jetzt aufzustellenden Sätze auf jede zwischen zwei solchen liegende Kugelschale anwenden). In einem solchen Medium ist jeder Strahl (ausgenommen die radial verlaufenden) eine stetig gekrümmte, symmetrische Kurve, welche an ihren beiden Endpunkten die Oberfläche unter gleichen Winkeln e trifft, und für deren Neigung und Krümmung folgende zwei einfache Sätze gelten, die aus der Theorie der "atmosphärischen Strahlenbrechung" schon lange bekannt sind ${ }^{1}$ ).

1. Ist $\mathrm{r}$ die Entfernung vom Kugelmittelpunkt, i der Neigungswinkel eines Strahles gegen den Radius, $\mathrm{v}$ die Strahlengeschwindigkeit $(=$ Wellengeschwindig. keit), so ist

$$
\frac{\mathbf{r} \sin \mathrm{i}}{\mathrm{v}}=\mathbf{c}
$$

wo c für jeden Strahl eine Konstante ist. Dieselbe kann entweder durch die Werte von $i$ und $v$ an der Oberfläche $(\overrightarrow{i,}, \vec{v})$, oder durch diejenigen von $r$ und $v$ im tiefsten Punkt des Strahles $\left(r_{m}, v_{m}\right)$, wo $i=90^{\circ}$ ist, ausgedrückt werden:

$$
c=\frac{R \sin \bar{i}}{\bar{v}}=\frac{r_{m}}{\nabla_{m}} . \quad(R=\text { Erdradius }) .
$$

2. Die Krümmung des Strahls, d. h. sein reziproker Krümmungsradius, bestimmt sich durch die Änderung der Geschwindigkeit mit $x$ und die Neigung $\mathrm{i}$ nach der Formel:

$$
\frac{1}{\rho}=\frac{1}{\mathrm{v}} \frac{\mathrm{d} \mathrm{v}}{\mathrm{dr}} \sin \mathrm{i}=\frac{\mathrm{c}}{\mathrm{r} \mathrm{d}} \mathrm{dr}
$$

dabei bedeutet ein positiver Wert von $\varrho$ eine gegen den Erdmittelpunkt hin konkave, ein negativer eine nach innen konvexe Krümmung. Dabei ist zu beachten, dass $\frac{1}{\mathrm{v}} \frac{\mathrm{dv}}{\mathrm{d} \mathrm{r}}=\frac{\mathrm{d} \log \mathrm{v}}{\mathrm{dr}}$ nur von $\mathrm{r}$ abhängt. Es ist hieraus ersichtlich, dass in derselben Tiefe (oder bei demselben $\mathrm{r}$ ) derjenige Strahl am stärksten gekrümmt ist, der horizontal verläuft, während für radiale Richtung natürlich die Krümmung verschwindet. Für die Krämmungen zweier verschiedener Strahlen, die unter verschiedenen Neigungen dieselbe Niveaufläche $r=$ const. durchsetzen, gilt die Proportion

$$
\frac{1}{\varrho_{1}}: \frac{1}{\varrho_{2}}=\sin i_{1}: \sin i_{2} \text {. }
$$

Da nun nach 7) $\sin i_{1}: \sin \dot{i}_{2}$ für zwei bestimmte Strahlen konstant ist, so gilt auch, wenn $i_{1}{ }^{\prime}, i_{2}{ }^{\prime}$ deren Neigungswinkel in einer anderen Tiefe $r^{\prime}$ sind:

11) $\quad \sin i_{1}{ }^{\prime}: \sin i_{2}{ }^{\prime}=\sin i_{1}: \sin i_{2}$ und auch zufolge 10):

$$
\varrho_{1}^{\prime}: \varrho_{2}^{\prime}=\varrho_{1}: \varrho_{2} \text {. }
$$

§ 7. Verwendung der Laufzeitkurvezur Bestimmung derStrablen.

Diese Sätze sind nun anzuwenden, um aus der "Laufzeitkurve einer bestimmten Wellenart (d. h. der 1. oder 2. Vorläufer) den Weg der entsprechenden Strahlen durch das Erdinnere mittelst Konstruktion oder Rechnang abzuleiten.

1) Eine einfache Ableitung findet sich z. B. bei WIEcrent, Gött. Nachr. 1907 , 19. 
Die "Laufzeitkurve" wird, wie S. 253 gesagt, erhalten, indem man die aus den Beobachtungen direkt zu entnehmenden Fortpflanzungszeiten als Funktion der längs der Erỏoberfläche gemessenen Entfernung $\triangle$ vom Epizentrum darstellt. Fig. 2 gibt eine Vorstellung von der Gestalt dieser Kurven für die ersten und zweiten Vorläufer nach WIECHERT und ZöPPRitz $(8,9)$. Diese Laufzeitkurven sind zwar nur aus drei Erdbeben: dem indischen vom 4. IV. 1905, dem kalabrischen vom 8. IX. 1905, dem kalifornischen vom 18. IV. 1906 abgeleitet, sind aber doch wegen der Art ihrer Berechnung wohl als genauer anzusehen, als die früher von Kövesligethy, Milne, Benndorf (7) und Otdham für die 1. Vorläufer aufgestellten, weil bei diesen teils Mittelwerte unter Mitbenutzung weniger sicherer Stationen verwendet, teils zur Ausgleichung die von ScHLÜTER angegebenen Einfallswinkel der Strahlen benutzt sind, welche ihrerseits aus den Amplitudenverhältnissen der vertikalen und horizontalen Schwingungskomponente an der Oberfläche ohne Berücksichtigung der Reflexion berechnet und daher, wie WIEchenT $(8, \S 12)$ gezeigt hat, fehlerhaft sind. Leider reichen die Zöppritz' schen Kurven nur bis zu $\triangle=11000 \mathrm{~km}$, da Beobachtungen in grösseren Entfernungen bei jenen 3 Erdbeben nicht vorlagen.

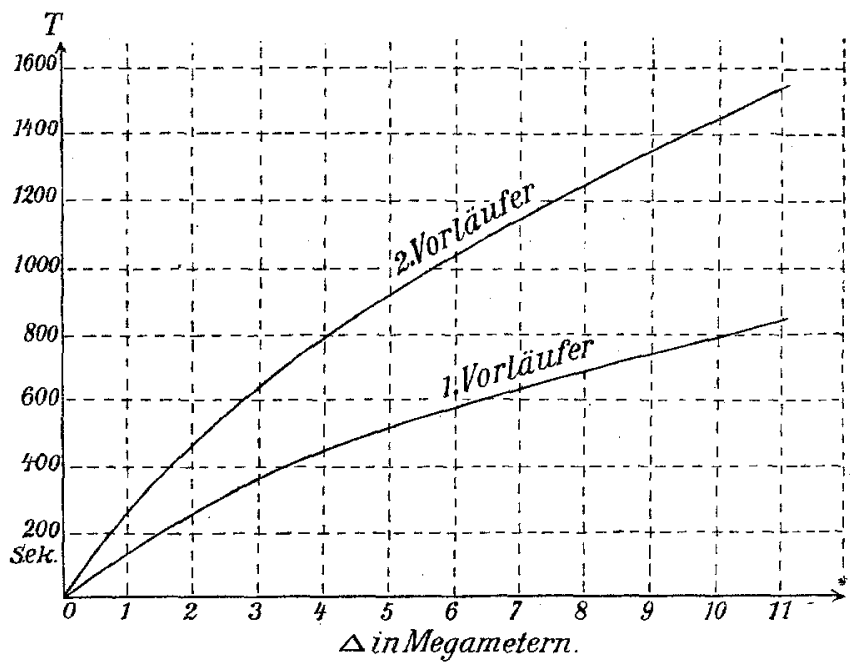

Fig. 2.

Die Laufzeitkurven der 1, und 2. Vorläufer (Fig. 2) zeigen einen sehr ähnlichen Verlauf in dem Sinne, dass die letztere durch Änderung des Ordinaten(Zeit-) Massstabes mit der ersteren fast zur Deckung gebracht werden kann. Beide Kurven sind stärker gekrümmt, oder steigen erst schneller, dann langsamer an, als es bei homogener Erde, also Fortpflanzung längs der Sehne mit konstanter Geschwindigkeit, der Fall sein würde.

Die Herdtiefe ist, wenn man nur die in grösserer Entfernung beobachteten Zeiten verwendet, vou keinem merklichen Einfluss auf die erhaltene Kurve, wie ZöPPRITZ (9) durch eine besondere Untersuchung gezeigt hat. (Nur sehr genauo Zeitbeobachtnngen in nächster Umgebung des Epizentrums würden einen solchen Einfluss erkennen lassen und damit auch zur Bestimmung der Herảtiefe dienen 
können.) Es darf daher im folgenden angenommen werden, dass der Herd in der Oberfläche selbst liege.

Die erste Folgerung, welche aus der Laufzeitkurve gezogen werden kann, betrifft die Einfallswinkel der Strahlen in ihrer Abhängigkeit von $\Delta$. Entnimmt man nämlich aus der Kurve den Neigungswinkel ihrer Tangente, so findet man dadurch (als dessen trigonometrische Kotangente) $\frac{\mathrm{d} \Delta}{\mathrm{d} T}$ oder die „scheinbare Oberfiächengeschwindigkeit $" \overline{\mathfrak{B}}$ der betreffenden Wellen; dies ist die Geschwindigkeit, mit

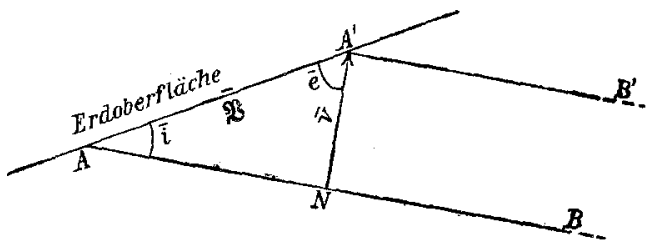

Fig. 3.

welcher die Durchschnittslinie einer Wellenfläche mit der Erdoberfläche längs der letzteren fortschreitet. Nan zeigt einge sehr einfache geometrische Betrachtung (siehe Fig. 3, worin $A B$ und $A^{\prime} B^{\prime}$ zwei im Zeitintervall 1 aufeinanderfolgende Lagen der an die Erdoberfläche stossenden Wellenfläche sind, also $\mathrm{NA}^{\prime}=\bar{\nabla}$, $A A^{\prime}=\overline{\mathfrak{B}}$ ist), dass zwischen dieser scheinbaren Geschwindigkeit $\overline{\mathfrak{B}}$ und der wahren Fortpflanzungsgeschwindigkeit $\bar{v}$ in der oberflächlichen Schicht die Beziehung besteht:

$$
\bar{\nabla}=\overline{\mathfrak{B}} \sin \overline{\mathrm{i}},
$$

so dass sich der „Einfallswinkel ${ }^{*}$ aus der Laufzeitkurve berechnen lässt mittelst der Formel:

$$
\sin \overline{\mathrm{i}}=\overline{\mathrm{v}} \cdot \frac{\mathrm{d} \mathrm{T}}{\mathrm{d} \Delta} .
$$

Allerdings wird, wegen der ungleichen Natur der obersten Erdschichten, der so berechnete Winkel nur dann den wahren Einfallswinkel an einem bestimmten Ort darstellen, wenn man für $\overrightarrow{\mathrm{v}}$ den gerade für die dort an der Oborfläche liegenden Gesteine geltenden Wert der Geschwindigkeit (longitudinaler oder transversaler) Wellen einsetzt, den man meist nicht genan kennt. Indessen kommt es für den jetzt betrachteten Zweck auch nicht auf den lokalen Wert von $\overline{\mathrm{i}}$ an, sondern auf seinen Mittelwert, den man richtig erhält, wenn man für $\overline{\mathrm{v}}$ den Mittelwert der Fortpflanzungsgeschwindigkeit der ersten bezw. zweiten Vorläufer in der äussersten Erdkruste setzt, der sich selbst aus den Beobachtungen von Nahbeben ableiten lässt (da ja bei geringer Epizentraldistanz die ankommenden Strahlen nur die oberflächlichen Schichten - bis zu Tiefen wenig grösser 'als die Herdtiefe durchlaufen haben).

Unter Annahme der Werte $\overrightarrow{\mathrm{v}}_{\mathrm{a}}=7,17$ und $\overline{\mathrm{v}}_{\mathrm{b}}=4,01 \mathrm{~km} / \mathrm{sec}$. fin den ZöPPRITZ and GEIGER folgende Werte von $i$. 


\begin{tabular}{|c|c|c|c|c|}
\hline \multirow[b]{2}{*}{$\begin{array}{c}\triangle \\
\text { (Megameter) }\end{array}$} & \multicolumn{2}{|c|}{ 1. Vorläufer } & \multicolumn{2}{|c|}{ 2. Vorläufer } \\
\hline & $\overline{\mathfrak{B}}_{\mathrm{a}}$ & $\mathrm{i}$ & $\overline{\mathfrak{B}}_{\mathbf{b}}$ & $\mathbf{i}$ \\
\hline 0 & 7,174 & $90^{\circ}$ & 4,01 & $90^{\circ}$ \\
\hline 1 & 7,66 & $69^{\circ} 18^{\prime}$ & 4,28 & $69^{\circ} 18^{\prime}$ \\
\hline 2 & 9,01 & $52^{\circ} 45^{\prime}$ & 5,04 & $52^{\circ} 45^{\prime}$ \\
\hline 3 & 10,95 & $40^{\circ} 57^{\prime}$ & 6,12 & $40^{\circ} 57^{\prime}$ \\
\hline 4 & 13,1 & $33^{0} 13^{\prime}$ & 7,43 & $32^{\circ} 40^{\prime}$ \\
\hline 5 & 15,7 & $27^{\circ} 12^{\prime}$ & 8,62 & $27^{\circ} 44^{\prime}$ \\
\hline 6 & 16,85 & $25^{0} 13^{\prime}$ & 8,77 & $27^{\circ} 12^{\prime}$ \\
\hline 7 & 17,2 & $24^{\circ} 42^{\prime}$ & 9,00 & $26^{0} 27^{\prime}$ \\
\hline 8 & 17,8 & $23^{\circ} 49^{\prime}$ & 9,35 & $25^{\circ} 24^{\prime}$ \\
\hline 9 & 18,65 & $22^{\circ} 38^{4}$ & 9,83 & $24^{\circ} 05^{\prime}$ \\
\hline 10 & 19,85 & $21^{\circ} 12^{\prime}$ & 10,47 & $22^{\circ} 31^{\prime}$ \\
\hline 11 & 21,4 & $19^{\circ} 37^{\prime}$ & 11,31 & $20^{\circ} 46^{\prime}$ \\
\hline
\end{tabular}

Man erkennt aus dieser Tabelle, dass schon in mässiger Entfernung die Oberflächengeschwindigkeiten sehr gross und die Einfallswinkel klein, also die Einfallsrichtungen (und zwar für beide Wellenarten) sehr steil werden (vergl. auch Fig. 5).

Aus den zusammengehörigen Werten von $\Delta$ und $\bar{i}$ müssen nun die entsprechenden Strahlenbahnen abgeleitet werden; sind diese gefunden, so kennt man auch $r_{m}$ und findet schliesslich mittelst der Relation 8) das zugehörige $v_{m}$, also insgesamt so viele zasammengehörige Wertepaare $\mathrm{v}$, r, als man Strahlen berechnet hat.

Die bisher zuverlässigte Lösung dieser Aufgabe rührt nun wiederum von WiecherT her und ist von ihm im Verein mit seinen Schülern ZöpprITz ( $\dagger$ ) und GETGER durchgeführt worden (8 a. 10). Die vorher von Milne (11), LÀska (6), OLDHaм (12) und KNotт (13) in dieser Richtung anternommenen Versuche sind teils deshalb verfehlt, weil zu spezielle Voraussetzungen (Zweiteilung der Erde in homogene Schale und homogenen Kern) gemacht wurden", teils weil die benutzten Laufzeitkurven zu ungenau waren; eine Untersnchung BENNNDORF's (7), in der ein Näherungsverfahren zur Lösung der Aufgabe für die ersten Vorläufer ohne spezielle Annahmen eingeschlagen wird, ergab ebenfalls keine zuverlässigen 'Resultate, weil dabei die irrtümliche ScHLÜTER'sche Bestimmung der Einfallswinkel $\overrightarrow{\mathrm{i}}$ zugrunde gelegt wurde.

Das WIECHERT'sche Verfahren ist ebenfalls ein Näherungsverfahren; es wird für die wirkliche Erde ein ans Kugelschalen von endlicher Dicke aufgebautes Medium substituiert, dabei aber den einzelnen Schalen nicht mehr konstante elastische Eigenschaften bezw. Geschwindigkeiten $v_{a}, v_{b} z u-$ geschrieben, sondern ein solches Gesetz ihrer Variation mit $r$, dass die Strahlen durchweg Kreisbögen werden. Die Bedingung hierfür ist zufolge Gleichung 9) dass $\frac{1}{r} \frac{d v}{d r}$ konstant ist; dann wird in der Tat dieser Gleichung zufolge für jeden Strahl $\rho$ konstant, also jedes innerbalb einer derartigen Kugelschicht 
verlaufende Strahlstück ein Kreisbogen. Die einzelnen Schichten müssen sich stetig aneinander schliessen, so dass beim Übergang von einer in die nächste die Strahlen keine Brechung, sondern nur eine sprungweise Änderung ibres Krümmungsradius erfahren. Natürlich kann man durch eine endliche Anzahl solcher KugelSchichten das wirkliche Gesetz der Änderung von $\mathrm{v}$ mit $\mathrm{r}$ und somit von $\mathrm{T}$ mit 4 nur approximativ darstellen, um so besser, je mehr und je dünnere Schichten man annimmt; es hat sich aber gezeigt, dass man schon mit sehr wenigen Schichten eine gute Annäherung erhält. Der besondere Vorzug dieser Approximationsmethode liegt nun darin, dass man die aus kreisbogenförmigen Stücken zusammengesetzten Strahlen sehr leicht durch Konstruktion oder Rechnung - unter Benutzung der Sätze 7 bis 12 - von der Erdoberfläche aus durch die einzelnen Schichten hindurch verfolgen kann.

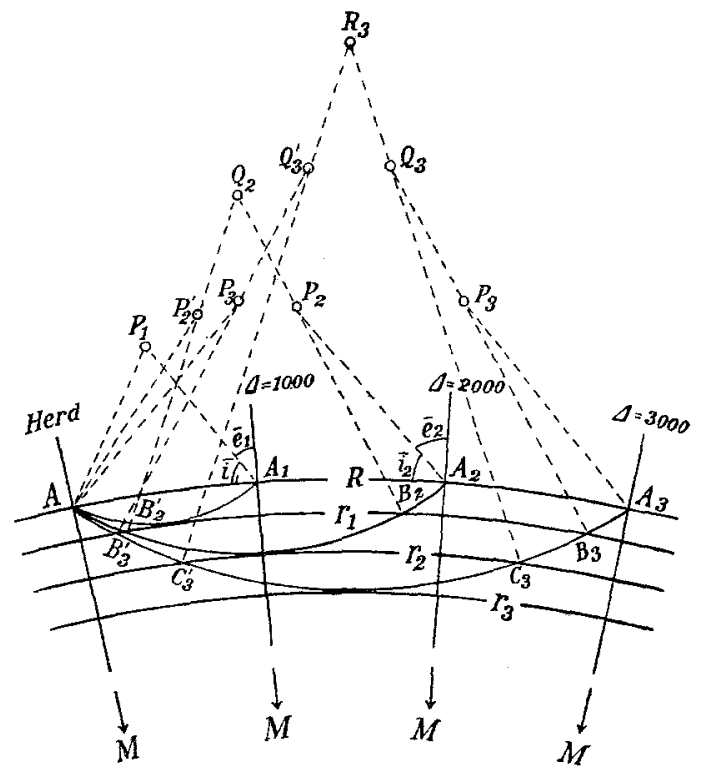

Fig. 4.

Dies wird nun etwa in folgender Weise durchgeführt. Man betrachtet zunächst einen Strahl, der in relativ kleiner Entfernung vom Herd, z. B. $\Delta=$ $1000 \mathrm{~km}$, wieder die Oberfläche erreicht und somit nur geringe Tiefen durchlaufen hat, als einheitlichen Kreisbogen ( $\mathrm{A} \mathrm{A}_{1}$ in Fig. 4) und kann für diesen, da man $j a$ seine Tangentenrichtungen in seinen Endpunkten $A$ und $A_{1}$ kennt, sofort den Mittelpunkt $P_{i}$ konstruieren, und damit auch den ganzen Strahl $\mathrm{A}_{A_{1}}$; man kennt also auch den Wert $r=r_{1}$ für seinen tiefsten Punkt, und diesen nimmt man als untere Grenze der ersten Schicht. Für einen zweiten Strahl, der etwa der Herddistanz $\triangle=A A_{2}=2000 \mathrm{~km}$ entsprechen möge, kennt man die Anfangsneigungen $\overline{\mathrm{i}}_{2}$ und folglich vermöge Gleichung 10) den Krümmungsradius seiner in der 1 . Schicht verlaufenden kreisbogenförmigen Stücke, kann also diese Stücke selbst sofort konstruieren; $P_{2}^{\prime}, P_{2}$ seien ihre Krümmungsmittelpunkte, $B_{2}{ }^{*}$ und $B_{2}$ die Punkte, wo sie die untere Grenze der 1. Kugelschicht er- 
reichen. Diese Punkte müssen nun durch einen Kreisbogen verbunden werden, der in ihnen die Tangentenrichtung mit den Bögen $\mathrm{A} \mathrm{B}_{2}{ }^{\prime}$ und $\mathrm{A}_{2} \mathrm{~B}_{2}$ gemein hat, und dessen Krümmungsmittelpunkt man als Schnittpunkt $Q_{2}$ der Verlängerungen von $B_{2}{ }^{\prime} P_{2}{ }^{\prime}$ and $B_{2} P_{2}$ findet. Bis zum tiefsten Punkt di eses Kreisbogens reehnet man nun die zweite Zone konstanter Strahlkrümmung. Für einen dritten, der Entfernung $\triangle=3000$ entsprechenden Strahl $\mathrm{AB}_{3}{ }^{\prime} \mathrm{C}_{3}{ }^{\prime} \mathrm{C}_{3} \mathrm{~B}_{3} \mathrm{~A}_{3}$ findet man wieder, ebenso wie zuvor für den zweiten Strabl, die Stücke $A_{3}{ }^{\prime}$ und $B_{3} A_{3}$ in der 1. Schicht, dann aus dem Einfallswinkel, nnter welchem diese die 2. Schicht

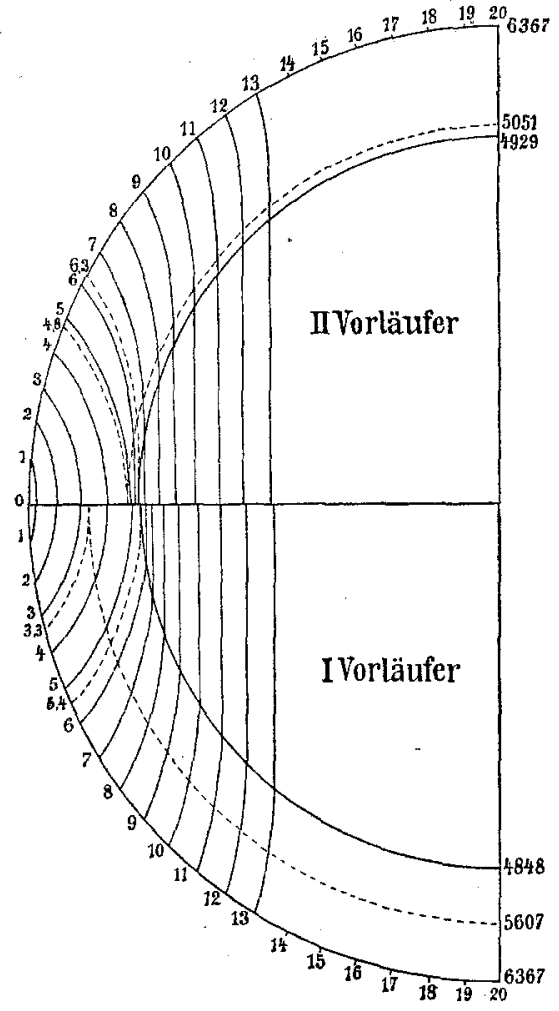

Fig. 5 . treffen, mit Benutzung von G], 10) die in der 2. Schicht liegenden Kreisbögen $\mathrm{B}_{3} \mathrm{C}_{3}{ }^{\prime}$ und $\mathrm{B}_{3} \mathrm{C}_{3}$, endlich wie oben den diese verbindenden, der 3 . Schicht angehörigen Kreisbogen $\mathrm{C}_{3}{ }^{\prime} \mathrm{C}_{3}$ und den. Wert $r_{m}=r_{3}$ für seinen tiefsten Punkt. In dieser Weise fortfahrend, kann man die Strahlen für immer grössere $\triangle$ konstruieren; für den tiefsten Punkt jedes so gefundenen Strahles liefert endlich Gl. 8) die zugehörige Geschwindigkeit. Fürr die zwischen den einzelnen Radien $\mathbf{r}_{m}$ liegenden $\mathbf{r}$-Werte ist $\mathbf{v}$ innerhalb jeder Schicht durch Integration der Gleichung $\frac{1}{r} \frac{d v}{d r}=$ const. zu berechnen. -

Natürlich kann das oben angegebene graphische Verfahren auch durchweg durch entsprechende Rechnung ersetzt werden, wobei grössere Genauigkeit zu erreichen ist. Diese Rechnung ist von dem früh verstorbenen K. ZÖPPRITZ für die 1. und 2. Vorläufer durchgefübrt und von L. GEIGER nach nochmaliger Durchrechnung kürzlich ausführlich veröffentlicht worden (10), nachdem provisorische Resultate schon 1907 von

WiEchenт $(8,9)$ mitgeteilt waren.

Bei der Durchrechnung kann sich herausstellen, dass mehrere der zunächst, wie in obigem Beispiel, willkürlich abgegrenzten Schichten „konstanter Krümmung " sich noch zu einer dickeren Schicht zusammenfassen lassen (z. B. würde dies für die Schichten (2) und (1) gelten, wenn die berechneten Punkte $P_{2}^{\prime}$ und $P_{2}$ sehr nahe mit dem Schnittpunkt $Q_{2}$ zusammenfielen); auch kann sich zeigen, dass die Grenzen zweckmässiger in anderen Tiefen, als sie den gerade gewählten Entfernungen $\triangle=1,2,3 \ldots$ entsprechen, anzunehmen sind. In der Tat ergab sich bei der Zöpprírz-GEIGER'schen Untersuchung, dass bis zu den untersuchlen Entfernungen mit. drei Zonen konstanter. Strahlkrümmung aus- 
zukommen war. Dabei ist überdies die erste gegen die zweite 7one nicht scharf abgegrenzt, so dass ihrex Grenze, die für die 1 . Vorläufer bei $760 \mathrm{~km}$, für die 2. Vorläufer bei $1316 \mathrm{~km}$ Tiefe angenommen wurde, offenbar keine physikalische Bedeutung zukommt. Anders ist es mit der unteren Grenze der zweiten Zone: es zeigte sich, dass dort, und zwar bei ca. $1520 \mathrm{~km}$ Tiefe für die ersten, bei $1400 \mathrm{~km}$ für die zweiten Vorläufer, die Strahlkrümmung sich sehr plötzlich ändert, so dass dieser Grenze also physikalische Realitat zuzuschreiben ist, wofür auch noch besonders die nahe Übereinstimmung der für die beiden Wellenarten gefundenen Tiefen dieser Grenzfläche spricht. Weiter zeigte sich, dass die unterhalb dieser Grenzen verlaufenden Strahlstücke merklich geradlinig sind, so dass also der „Kern“ der Krde, wenigstens bis zu den Tiefen, die von den hier berechneten Strahlen erreicht wurden, als merklich h o mog e n anzusehen wäre.

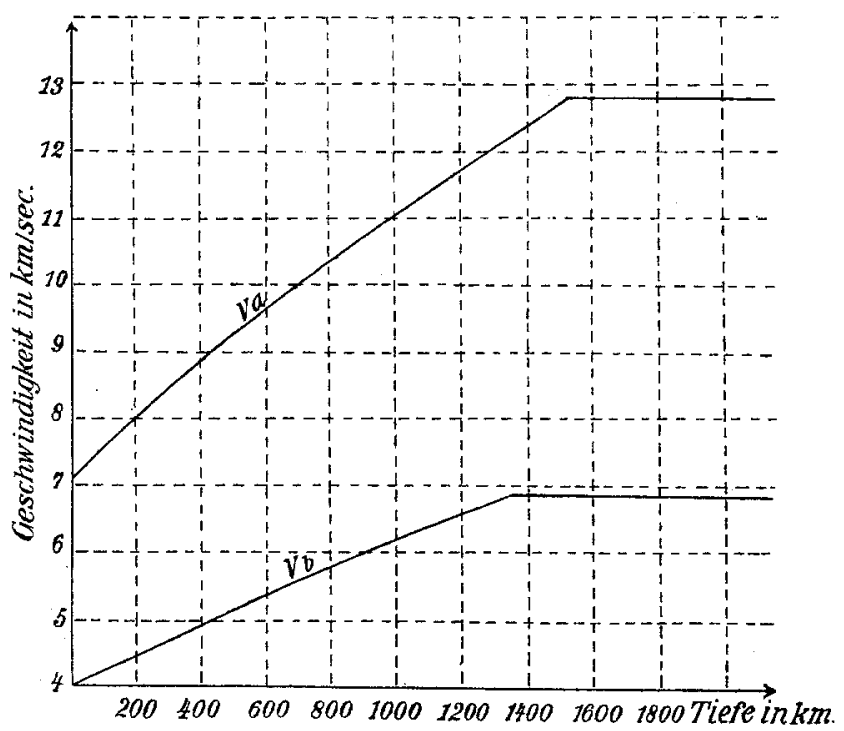

Fig. 6.

Den wirklichen Verlauf der Strahlen stellt hiernach Fig. 5 dar (die eine Hälfte für die ersten, die andere für die zweiten Vorläufer); die punktierten Strahlen sind diejenigen, welche gerade die obigen Zonengrenzen berühren; die punktierten Kreise stellen die nur zum Zweck der Rechnung eingeführte Grenze der 1. und 2. Zone dar, die ausgezogenen Kreise (den Kugelfächen von 4848 bezw. $4929 \mathrm{~km}$ Radius entsprechend) die Grenze des Kernes, wie sie sich aus den beiden Wellenarten ergibt. Wie man sieht, findet hiernach an der Grenze des Kernes keine Brechung der Strahlen statt; wenigstens kann eine solche, wenn vorhanden, nur sehr gering sein, so dass sie durch die ausgeführte Strahlberechnung nicht nachweisbar ist. Die Änderung der Geschwindigkeiten $v_{a}$ und $v_{b}$ mit der Tiefe bis zum Kern hin, wie sie nun schliesslich in der oben S. 254 angegebenen Weise berechnet werden konnte, ist in nachstehender Tabelle zusammengestellt und in Fig. 6 graphisch veranschaulicht. Man sieht, dass beide Geschwindigkeiten nahezu 
linear von den Werten 7,17 bezw. $4,00 \mathrm{~km} / \mathrm{sec}$, die sie in den oberflächlichen Schichten besitzen, bis zu den an der Grenze des Kerns erreichten und von da an zunächst konstant bleibenden Werten 12,78 and 6,87 ansteigen.

\begin{tabular}{c|c|c|c}
\hline \hline Tiefe (km) & $\mathbf{r}(\mathbf{k m})$ & $\begin{array}{c}\mathbf{v}_{\mathbf{a}} \\
(1 . \text { Vorläufer }) \\
\mathrm{km} / \mathrm{sec} .\end{array}$ & $\begin{array}{c}\mathbf{v}_{\mathbf{b}} \\
(2 . \text { Vorläufer }) \\
\mathrm{km} / \mathrm{sec} .\end{array}$ \\
\hline \hline & & & \\
\hline 100 & 6367 & 7,17 & 4,00 \\
200 & 6267 & 7,60 & 4,24 \\
400 & 6167 & 8,01 & 4,47 \\
600 & 5967 & 8,83 & 4,93 \\
800 & 5767 & 9,62 & 5,37 \\
1000 & 5567 & 10,37 & 5,80 \\
1200 & 5367 & 11,07 & 6,21 \\
1400 & 5167 & 11,75 & 6,60 \\
1430 & 4967 & 12,40 & 6,87 \\
1500 & 4937 & - & 6,87 \\
1519 & 4867 & 12,72 & - \\
& 4848 & 12,78 & -
\end{tabular}

Dass diese Konstanz der Geschwindigkeiten im Kern bis zum Mittelpunkt der Erde hin gelten sollte, erschien von vornherein allerdings sehr unwahrscheinlich und ist auch nach einex Bemerkung WiEchents $(8$, S. 94) bereits widerlegt durch die Beobachtung der Laufzeiten eines Erabebens, welches 1907 in der Nähe von Samoa stattfand; die Laufzeiten der Strahlen bis Göttingen, welche Tiefen von ca. $3000 \mathrm{~km}$ erreicht haben müssen, waren erheblich grösser, als sie nach der obigen Annahme hätten sein müssen, woraus zu schliessen ist, dass gegen den Erdmittelpunkt hin wieder eine $A b n a h m e$ der Geschwindigkeiten va und $v_{b}$ eintritt - nach WIEcherTs Schätzung vielleicht bis auf $10 \mathrm{bezw} .6 \mathrm{~km} / \mathrm{sec}$. Genaueren Aufschluss hierüber werden erst künftige Untersuchungen von Erdbeben, die über nahe den halben Erdumfang verfolgt werden können, liefern ${ }^{1}$ ).

8 8. Deutung der gewonnenen Resultate.

Am interessantesten an den vorstehend berichteten krgebnissen ist ohne Zweifel der Nachweis der plötzlichen Veränderung derphysikalischen Beschaffenheit des Erdinnern in einer Tiefe von rund $1500 \mathrm{~km}$ unter der Oberfläche, d. i. etwas weniger als $1 / 4$ des Erdradius. Denn dieses Resultat kann in gewissem Grade als Bestätigung der von WIECHERT (1) schon 11 Jahre früher auf anderem Wege (siehe oben, S. 250) erschlossenen Zusammensetzung der Erde aus einer Gesteinsschale von 1300 bis $1600 \mathrm{~km}$ Dicke und einem

$\left.{ }^{1}\right)$ Kürzlich hat H. Batemáñ (Physik. Ztschr. 11, S. 96-99, 1910) durch eine Berechnung, die sich auf eine empirische Näherungsformel für die Micneschen Laufzeiten gründet, ebenfalls eine (geringe) Wiederabnahme von $v_{a}$ gegen den Erdmittelpunkt hin gefunden, im übrigen aber einen wesentlich anderen Verlauf von $v_{a}$ als WIECHERT. 
Metallkern gelten. Allerdings nur in gewissem Grade: denn bei jener Borechnung hatte WIECHERT dem Kern sowohl, als auch dem Gesteinsmantel homogene Dichte zugeschrieben. Nun weisen die gefundenen Werte der Geschwindigkeiten $\mathrm{va}_{a}$ und $\mathrm{v}_{\mathrm{b}}$ allerdings (wenn wir von der noch unsicheren Wiederabnahme in den inneren Teilen des Kerns absehen) auf Homogenität des Kernes hin ${ }^{1}$; aber ihre starke Veränderung in der Schale lässt es höchst unwahrscheinlich erscheinen, dass diese konstante Dichte besitzen sollte. Dazu kommt die bemerkenswerte Tatsache des merklich stetig e n Überganges der Fortpflanzungsgeschwindigkeiten an der Grenzfläche des Kernes. Diese macht es in hohem Grade unwahrscheinlich, dass diese Grenzfläche eine wirkliche Unstetigkeitsfläche für die physikalischen Eigenschaften (insbesondere die Dichte) und die chemische Zusammensetzung der Erdsubstanz sei, und spricht vielmehr sehr zugunsten der Vorstellung, dass auch die stoffliche Natur des Gesteinsmantels stetig - etwa durch immer mehr zunehmende Beimischung von metallischem Eisen, wie wir sie ja an Meteoriten kennen - in diejenige des Metallkerns übergeht. Die "Grenzfläche des Kernes" hätte danach nur die Bedeutung der Grenze, bei welcher die Substanz merklich reinmetallisches Eisen (oder Nickeleisen) geworden ist, und an der daher die Änderung ihrer physikalischen Eigenschaften zunächst plötzlich aufhört.

Mit anderen Worten: die Grenze von "Kern" und, Mantel“ der Erdeist nichteine Unstetigkeitsflächefür diephysikalische und chemische Beschaffenheit selbst, sondern nur für deren Änderung mit der Tiefe.

Die Wiederabnahme der Erdbebenstrahlgeschwindigkeiten gegen das Zentrum des Kernes lässt sich durch Zunahme der Dichte, sei es durch den wachsenden Druck, sei es - was wahrscheinlicher ist - durch wachsende Beimischung schwererer Metalle, leicht erklären. Es sei jedoch bemerkt, dass vorstehendes nicht ganz den Schlussfolgerungen entspricht, zu welchen WIECHERT selbst gelangt ist. Derselbe neigt vielmehr zu der Vorstellung (3, S. 46), dass die Grenzfläche doch eine wirklich $\theta$ Unstetigkeitsfläche sei, und dass der stetige Übergang der v-Werte an der Grenzfläche auf einer Gesetzmässigkeit des elastischen Verhaltens aller Materie unter sehr hohem Druck (Abhängigkeit der Elastizität von den Atomen allein, und Proportionalität des "elastischen Widerstandes der Atome" mit ihrer Masse) beruhe. -

Um aus den gefundenen Zahlwerten der Geschwindigkeiten $v_{a}$ und $v_{b}$ weitere Schlüsse zu ziehen, ist daran zu erinnern, dass nach Gl. 1) und 2) diese Geschwindigkeiten, ausser von den Elastizitätskonstanten, von der Dichtigkeit der Substanz abhängen; und da wir das Gesetz der Dichtigkeitsverteilnng im Gesteinsmantel nicht kennen, so können die klastizitätskonstanten $a^{2}$ und $b^{2}$ selbst für diesen $\mathrm{nicht}$ berechnet werden. Wohl aber kann aus $v_{a}: v_{b}$ ihr Verhältnis, und damit auch (zufolge Gl. 4) die PoIsson sche Konstante $\mu$ berechnet werden. Ein Auszug aus den hierfür von GEIGER (10) gefundenen Werten für verschiedene Tiefen folgt hier:

1) Mit Sicherheit kann auch aus konstanten Werten von $v_{a}$ und $v_{b}$ nicht auf Homogenität geschlossen werden, da nach den Formeln 1) und 2) deren Konstanz auch mit veränderlichen Elastizitätskonstanten und veränderlicher Dichto vereinbar ist, sofern nur erstere sich proportional mit der letzteren ändern. 
Besprechungen.

$\begin{array}{cc}\text { Tiefe in } \mathrm{km} & \mu \\ 0 & 0,2578 \\ 100 & 0,2732 \\ 200 & 0,2724 \\ 400 & 0,2736 \\ 600 & 0,2737 \\ 800 & 0,2724 \\ 1000 & 0,2704 \\ 1200 & 0,2685 \\ 1400 & 0,2795 \\ (1500 & 0,297)\end{array}$

Man sieht, dass $\mu$ in allen Tiefen im Gesteinsmantel wenig grösser als $1 / 4$ jst, d. h. als derjenige Wert, der sich aus der molekularen Theorie der Elastizität unter der Annahme, dass die elastischen Molekularkräfte Zentralkräfte sind, für isotrope Körper ergibt.

Für den Erdkern kann man, da seine Dichte mit ziemlicher Wahrscheinlichkejt zu etwas über 8 angenommen werden darf, auch die Werte der Elastizitätskonstanten selbst annähernd berechnen und findet nach der Formel 5) eine Kompressibilität, die etwa 5 mal kleiner ist, als die von Stahl bei gewöhnlichen Temperaturen und Drucken; ebenso ist die Starrheit etwa $4^{3} / 4$ mal grösser als die des Stahles ${ }^{1}$ ). Beides ist, da der Kern höchet wahrscheinlich wesentlich aus Eisen besteht, dem ungeheueren Drucke zuzuschreiben.

Hiermit sind die bisher aus den seismischen Beobachtungen zu ziehenden Schlüsse über die physikalische Natur des Erdkörpers erschöpft. Wenngleich dieselben vielleicht noch manche Modifikation erfahren werden, so wird man doch zugestehen müssen, dass das Erreichte in Anbetracht der Jugend der exakten Seismologie schon viel ist, und wird sicher erwarten dürfen, dass bei fortschreitender Vermehrung und Verfeinerung des Beobachtungsmaterials noch reiche Aufschlüsse über die physikalische Natur und den Bau des Erdkörpers; sowohl in seinem tiefsten Innern, als in der äusseren Rinde, zu gewinnen sein werden.

\section{Zur Geologie des zentralen Ost-Afrika.}

Von F. Krenkel, München.

(Schluss.)

In der Breite des Magad-Sees beginnt der zweiseitige Graben, nachdem auch die südlich von ihm in einzelne Stufen und Horste völlig aufgelöste und unzusammenhängende Ostwand Geschlossenheit gewonnen hat (59, S. 498). Auf britischem Gebiet bis zum Naivasha-See erlangt der Graben eine grosse Einheitlichkeit und Enge, so dass wirklich der Eindruck einer schmalen, von steilen Bruchrändern flankierten Einsenkung hervorgerufen wird. Sein Westrand zejgt zunächst am Magad eine merkwürdige Stufenbildung (59, S. 485), indem ihm westlich

1). Hierbei sind für Stahl die Werte $\mathrm{E}=21000 \frac{\mathrm{kg} \text {-Gew. }}{\mathrm{mm}^{2}}, \mu=0,28$ angenommen. 